\title{
Thanks to reviewers in 2021
}

\section{Nicola Oberbeckmann-Winter ${ }^{1}$}

Accepted: 21 September 2021 / Published online: 14 October 2021

(C) Springer-Verlag GmbH Germany, part of Springer Nature 2021

The mission of Analytical and Bioanalytical Chemistry $(\mathrm{ABC})$ is the communication of excellent research work from all fields of (bio-)analytical chemistry. Clearly, a scientific journal's greatest responsibility toward the scientific community is to ensure that all contributions accepted for publication are rigorously but fairly reviewed in a timely manner. For this reason, all manuscripts published in $\mathrm{ABC}$ are reviewed by expert referees. The final quality of papers accepted for publication depends to a considerable extent on the reviewers, who give valuable constructive criticism to the authors and guidance to the editors. The input and dedication of all our reviewers are therefore highly appreciated by editors, authors and readers of the journal.

At the end of 2021, we gratefully acknowledge the valuable support of the following scientists who have reviewed papers for the journal during the past 12 months:

\author{
Abad-Fuentes, A \\ Abd Karim, NH \\ Abdelhamid, HN \\ Ackerman, B \\ Actis, $\mathrm{P}$ \\ Adami, G \\ Adams, A \\ Adelani, $\mathrm{P}$ \\ Adıgüzel, $Y$ \\ Adriaens, $\mathrm{M}$ \\ Afonso, $\mathrm{C}$ \\ Afshar Mogaddam, MR \\ Aguilar-Caballos, MdlP \\ Aguirre Pastor, MÁ \\ Ajayi, F \\ Alarcón Ángeles, V \\ Albert, J \\ Albertí, J \\ Ali, I \\ Aliaño-González, MJ \\ Alijanianzadeh, M \\ Altmann, F \\ Alygizakis, NA \\ Amoresano, A
}

\author{
Anderson, CE \\ Anderson, JL \\ Andersson, JT \\ Anderton, C \\ Andrade Garda, JM \\ Andreescu, $\mathrm{S}$ \\ Andrén, LE \\ Anfossi, L \\ Ángel Rodríguez-Delgado, M \\ Antico, E \\ Antoine, $\mathrm{R}$ \\ Antonova, S \\ Aprea, E \\ Arce, L \\ Ariese, F \\ Arigò, $\mathrm{A}$ \\ Armenta, S \\ Armstrong, DW \\ Arsene, C \\ Avino, $\mathrm{P}$ \\ Awad, D \\ Azimzadeh, M \\ Babakov, VN
}

Badea, M

Badhulika, S

Badía, R

Baggiani, C

Bagheri, $\mathrm{H}$

Bagheri, Y

Baker, C

Baker, E

Baker, LA

Bakirhan, NK

Bala, C

Baldini, F

Baldrich, E

Bamba, T

Banoub, J

Bao, $N$

Barderas, $\mathrm{R}$

Barding, G

Barek, J

Barrios, CA

Bartlett, MG

Bartosik, M

Baser, $\mathrm{H}$

Bayliss, M

Nicola Oberbeckmann-Winter

nicola.oberbeckmann-winter@ springer.com

1 Analytical and Bioanalytical Chemistry, Springer Verlag,

Tiergartenstrasse 17, 69121 Heidelberg, Germany 
Beach, DG

Beauchamp, J

Becher, F

Beklemishev, M

Belder, D

Beljebbar, A

Bendell, LI

Benito Peña, E

Bennett, A

Benskin, JP

Bergquist, J

Berlina, AN

Bermejo Barrera, $\mathrm{P}$

Berti, F

Bertoncello, $\mathrm{P}$

Bertotti, M

Bertrand-Michel, J

Bettmer, J

Beuerle, T

Bezerra, G

Bi, X

Bicchi, C

Bilewicz, R

Birch, B

Blanco-López, MC

Blum, M-M

Bocklitz, T

Bocková, M

Boczkaj, G

Boggs, ASP

Bongiorno, D

Bonhommeau, S

Borges, DLG

Borik, A

Borrull, F

Borsdorf, $\mathrm{H}$

Bose, K

Bossi, AM

Brajter-Toth, A

Brandi, J

Breidbach, A

Brett, CMA

Brodbelt, J

Broothaerts, W

Buchberger, W

Buckley, B

Bunk, DM

Bunkoed, O

Burkin, MA

Burmistrova, NA

Buttinger, $\mathrm{G}$

Byrne, HJ
Čabala, R

Cacciola, F

Cagliero, C

Cahill, JF

Cai, Q

Calvano, CD

Camara, JE

Cameron, S

Campuzano, S

Can, NO

Canela-Garayoa, R

Cannazza, G

Cantafora, AD

Cao, Q

Capitan-Vallvey, LF

Cappa de Oliveira, LF

Capriotti, AL

Cardeal, ZL

Cárdenas Aranzana, S

Carere, M

Careri, M

Carinhanha Caldas Santos, J

Carrasco, $\mathrm{S}$

Carro, AM

Carter, C

Carvalho, LF

Caselli, F

Castro, $\mathrm{K}$

Cataldi, TRI

Catani, N

Cauerhff, A

Causon, $\mathrm{T}$

Cavalier, E

Cavaliere, $\mathrm{C}$

Cavallaro, G

Cawley, AT

Cela Torrijos, R

Cerrato, A

Chai, W

Chai, Y-Q

Chailapakul, O

Chaix, C

Chansiri, K

Chaurand, P

Chen, B

Chen, $\mathrm{H}$

Chen, J

Chen, L

Chen, S

Chen, T-H

Chen, W

Chen, Z
Cheng, G

Chinnambedu Murugesan, R

Chiu, T-C

Cho, TJ

Choi, H-K

Choi, JR

Christopoulos, T

Chung, SWC

Citterio, D

Clark, KD

Clarke, $\mathrm{N}$

Claude, B

Clench, MR

Cleveland, $\mathrm{M}$

Cobice, D

Coelho, C

Cologna, SM

Colombini, MP

Colon, L

Coltro, W

Combès, A

Concheiro-Guisan, M

Cong, $\mathrm{H}$

Conklin, SD

Cooks, RG

Cooper, $\mathrm{H}$

Corbisier, $\mathrm{P}$

Corti, C

Costa-Fernández, JM

Covey, $\mathrm{T}$

Cox, HD

Crimmins, BS

Cristea, CV

Cuccia, L

Culbertson, CT

Culha, M

Cunha, S

Cvačka, J

Dai, X

Dallas, D

Dang, F

Daniele, S

Danielson, N

Dannenberger, D

David, F

Davies, SR

Davoli, E

de Almeida, MP

de los Santos Álvarez, N

De Pauw, E

Deckert, V 
Dedieu, M

Delatour, V

DeMarco, ML

Demeke, $\mathrm{T}$

Deng, B

Deng, C

Deng, $\mathrm{H}$

Deng, Q

Depciuch, J

Deredge, D

Desaire, $\mathrm{H}$

Desire, C

Devonshire, AS

Dhamane, S

Díaz-García, ME

Dickert, FL

Dietrich, $\mathrm{R}$

Dimandja, J-MD

Dimartino, $\mathrm{S}$

Dina, $\mathrm{N}$

Ding, C-F

Ding, $S$

Ding, $Z$

Dixon, D

Do, TD

Dobnik, D

Dodds, J

Donato, $\mathrm{P}$

Dong, $\mathrm{C}$

Dong, Y

Donnarumma, D

D'Orazio, G

Dornellas, FM

Dotsikas, Y

Dou, $\mathrm{H}$

Downard, K

$\mathrm{Du}, \mathrm{J}$

Dubey, DK

Duewer, D

D'Ulivo, A

Dunn, WB

Dürkop, A

Dutra, RF

Dzantiev, BB

Eberlin, LS

Eichmann, T

El Hadri, H

El-Ahmady, S

Elbadry, N

Ellis, SR

El-Maghrabey, MH

Emons, $\mathrm{H}$
Emteborg, H

Enders, J

Ermini, ML

Eskelin, KJ

Esteves da Silva, JCG

Fakhrullin, RFU

Fanali, C

Fanizzi, FP

Farahani, A

Farka, Z

Fattorini, $\mathrm{P}$

Faulkner, D

Favretto, D

Fechner, $\mathrm{P}$

Fei, J

Feng, J

Feng, L

Feng, W

Feng, Y-Q

Fernanda Silva, M

Fernandez, JA

Fernandez-Alba, AR

Fernandez-Arguelles, MT

Fernandez-Lima, F

Fernández-Sánchez, JF

Ferreira, CR

Firdaus, ML

Fletcher, JS

Flores, EMdM

Fontanals, N

Forbes, $\mathrm{P}$

Foret, F

Foster Mesko, MF

Franco, R

Frascione, N

Fratila, RM

Frolov, A

Frosch, T

Fu, Q

$\mathrm{Fu}, \mathrm{Z}$

Fujii, S-I

Gai, F

Gallardo, E

Gallello, G

Galli, R

Gamella, M

Gao, P

Gao, Q

Gao, X

Garcia, CD

Garcia, R
García-Barrera, T

Garcia-Villalba, R

Gardiner, PHE

Gargallo, R

Garrard, K

Garrido, M

Garris, $\mathrm{P}$

Garvey, J

Gasparrini, F

Ge, G

Ge, J

Genc, R

Gentili, A

Giannetti, A

Gibson-Daw, G

Gigante, GE

Gilmore, I

Gimenez-Romero, C

Gionfriddo, E

Givelet, L

Goenaga-Infante, $\mathrm{H}$

Goldman, ER

Gomez, M

Gomez Ariza, JL

Gomila, RM

Gong, M

Gonzales Lopez, LJ

Gonzalez-Rodriguez, J

Gonzato, C

Goodpaster, JV

Gorinstein, S

Goto-Inoue, N

Grant, E

Grasso, G

Greener, J

Greguš, M

Grigoryev, A

Gröger, TM

Groseclose, MR

Gross, JH

Guan, F

Guan, Y

Guo, L

Guo, M

Guo, Y

Guo, Z

Gupta, R

Guttman, A

Guyon, F

Hackett, W

Hackl, T

Haldemann, C 
Hamm, G

Han, D

Hang, W

Hanrieder, J

Harris, J

Harris, R

Harvey, CJ

Hasanzadeh, M

Hashemi, P

Hassan, U

Haupt, K

Hauser, PC

Hawkridge, A

Hayden, $\mathrm{O}$

Hayen, $\mathrm{H}$

Hayes, MA

$\mathrm{He}, \mathrm{H}$

He, L

$\mathrm{He}, \mathrm{Y}$

Heberger, K

Hecht, E

Heck, A

Hedeland, M

Heijs, B

Heinzen, $\mathrm{H}$

Heise, HM

Heiss, C

Helander, A

Henry, C

Henry, S

Herkendell, K

Hermabessiere, L

Hernandez, F

Hernández Borges, $\mathrm{H}$

Hernandez-Cordoba, M

Hernández-Mesa, M

Herr, A

Herrero, M

Hess, N

Heudi, O

Hicks, L

Hildebrandt, N

Hilder, E

Hill, N

Hinrichs, K

Hirai, H

Ho, K-S

Hohberger, B

Holčapek, M

Holland, LA

Hollender, J

Hongsibsong, S

Hoppe, M
Horvai, G

Hou, Y

Hsu, FF

Hsu, P

$\mathrm{Hu}, \mathrm{B}$

$\mathrm{Hu}, \mathrm{J}$

$\mathrm{Hu}, \mathrm{Q}$

Hu, W

$\mathrm{Hu}, \mathrm{Y}$

Huang, CZ

Huang, G

Huang, RY-C

Huber, CG

Hurth, C

Ifa, DR

Igne, B

Iniesta, J

Iqbal, N

Isobe, $\mathrm{T}$

Itoh, $\mathrm{T}$

Ivarsson, Y

Ivleva, NP

Jackson, SN

Jaffrezic-Renault, N

Jahouh, F

Jährmann, A

Jakubowski, N

Jankowski, KJ

Janssen, H-G

Jemere, A

Ji, H

Jiang, H

Jiang, J

Jiang, $\mathrm{T}$

Jiang, $\mathrm{X}$

Jiang, Y

Jie, G

Jin, $\mathrm{T}$

Jin, Y

Jing, $\mathrm{T}$

Jochmann, MA

John, H

John, SA

Johnson, M

Johnson, RC

Johnson-Restrepo, B

Johnston, L

Jung, J

Junot, C

Junqueira, M

Jurado Sánchez, B
Kadimisetty, K

Kafouris, D

Kailasa, SK

Kaji, N

Kakarla, RR

Kaljurand, M

Kalogianni, DP

Kaltashov, IA

Kameyama, A

Kandil, MM

Kang, T

Kara, P

Karlsson, N

Karst, U

Kato, M

Katz, E

Kaufmann, A

$\mathrm{Ke}, \mathrm{R}$

$\mathrm{Ke}, \mathrm{S}$

Keithley, RB

Kéki, S

Kellogg, J

Kennedy, RT

Kessler, RW

Keyes, TE

Kiefer, J

Kim, B

Kim, S

Kintzios, S

Kinumi, T

Klampfl, CW

Kneipp, J

Knepper, TP

Knochenmuss, $\mathrm{R}$

Knopp, D

Koch, M

Köfeler, H

Kohlheyer, D

Konermann, L

Kong, KV

König, S

Kosman, J

Kötting, C

Kramer, M

Kranz, C

Krauss, M

Krska, R

Kruss, S

Krylov, SN

Kuban, P

Kuesters, M

Kuklenyik, Z

Kumar, B 
Kumar, D

Kumar, JR

Kumari, S

Kuo, T-R

Kutter, JP

Laemmerhofer, M

Laganà, A

Laiwattanapaisal, W

Lambert, C

Lammertyn, J

Lamminmäki, U

Lan, $\mathrm{M}$

Lanças, FM

Landero, JA

Lanekoff, I

Lang, BE

Lao, W

Lauth, J

Lavine, BK

Lebert, D

Lebrilla, C

Lee, HK

Lee, LC

Lee, Y-J

Legnaioli, S

Lehmann, WD

Lehotay, SJ

Leng, J

Leopold, $\mathrm{N}$

Lesur, A

Li, B

Li, D

Li, F

Li, G

Li, HY

Li, J

Li, L

Li, N

Li, P

Li, S

Li, T

Li, X

$\mathrm{Li}, \mathrm{X}(\mathrm{J})$

Li, Y

Li, Y-F

Li, Z

Lian, H-Z

Liang, $\mathrm{Y}$

Lieberzeit, PA

Lin, C

Lin, $\mathrm{S}$

Lin, Z
Linden, $\mathrm{R}$

Linz, $\mathrm{T}$

Lisdat, $\mathrm{F}$

Litescu, SC

Liu, B

Liu, J

Liu, K

Liu, L

Liu, Q

Liu, $\mathrm{S}$

Liu, $X$

Liu, Z

Llompart, $\mathrm{M}$

Lobo-Castañón, MJ

Locatelli, M

López Molinero, Á

Lopez-Sanchez, JF

Lou, X

$\mathrm{Lu}, \mathrm{H}$

$\mathrm{Lu}, \mathrm{J}$

$\mathrm{Lu}, \mathrm{L}$

Lu, X

Lucena Rodríguez, $\mathrm{R}$

Lundberg, $\mathrm{P}$

Luo, $X$

Luppa, PB

Luptakova, D

Lv, Y

Ma, J

Ma, L

Mace, CR

MacManus-Spencer, LA

Madeddu, R

Madrakian, $\mathrm{T}$

Maekawa, N

Magi, E

Maheshwari, V

Mahmud, I

Mairinger, $\mathrm{T}$

Maiwald, M

Malek, K

Malhotra, VD

Malinovskiy, DN

Malitesta, C

Maloney, $\mathrm{T}$

Mani, V

Mansour, FR

Mao, L

Maquieira, Á

Marcelli, A

Marchegiani, E

Marco, M-P
Marcus, RK

Marina, ML

Mark, KJ

Marken, F

Markin, AV

Marshall, J

Martin, FL

Mashile, GP

Masselon, C

Matejčík, $\breve{S}$

Matousek, P

Matraszek-Zuchowska, I

Matsuda, Y

Mattarozzi, M

May, JC

Maya Alejandro, F

Mayboroda, OA

Mayer, M

Mayerich, D

Mayr, T

Mazumder, A

McAdam, KG

McCauley, JW

McCord, BR

McDonnell, L

McMillan, NJ

Meagher, RJ

Mechler, A

Mechref, Y

Medana, C

Meija, J

Meixner, A

Melanson, JE

Men, D

Meng, L

Mesquita, $\mathrm{R}$

Mester, Z

Miao, $P$

Micalizzi, G

Michael, AC

Michalke, B

Micheli, L

Michelini, E

Migaud, M

Mikhailidi, A

Milavec, M

Milea, D

Miller, K

Min, J

Min, JZ

Minteer, $S$

Miranda Castro, R

Miró, M 
Misal, SA

Mishra, A

Misra, BB

Mitchell, J

Mnatsakanyan, M

Möder, M

Mohammadnejad, L

Mol, HGJ

Molina Diaz, A

Molinié, R

Mollarasouli, F

Monaci, L

Mondello, L

Moon, MH

Moore, DS

Moorthy, GS

Morais, $\mathrm{S}$

Morini, L

Morlock, GE

Mormann, M

Moscone, D

Motaharian, A

Moyano, E

Mravcova, L

Mudalige, $\mathrm{T}$

Muddiman, DC

Müller, T

Muneeruddin, $\mathrm{K}$

Nan, J

Nayak, P

Neef, SK

Nehmé, R

Nemutlu, E

Nerin de la Puerta, C

Neubert, $R$

Neusü $\beta, C$

Nguyen, B

Nicoli, R

Nielen, $\mathrm{M}$

Niessner, $\mathrm{R}$

Nikiforidis, C

Nilsson, L

Nitride, C

Nivelle, $\mathrm{M}$

Nordborg, A

Nordin, G

Nowak, S

Nugen, SR

Nunes, RSM

O'Rourke, M

O'Connor, G
Ogrinc, N

Oh, HB

Oliveira, HM

Oprea, A

Oßmann, BE

$\mathrm{Ou}, \mathrm{Y}$

Ouyang, G

Ozalp, VC

Ozkan, SA

Pabst, N

Paczkowski, S

Pagel, K

Paglia, G

$\mathrm{Pal}, \mathrm{S}$

Palacio Lozano, DC

Palchetti, I

Pallaoro, A

Palleschi, V

Palzer, S

Panderi, IE

Panne, U

Pappas, D

Parastar, H

Park, JP

Park, KS

Park, TJ

Paseiro Cerrato, R

Patra, D

Patterson, NH

Paul, R

Paulino, BN

Paull, B

Pedrero, M

Peeters, $\mathrm{M}$

Peña-Farfal, CG

Penkov, $\mathrm{S}$

Pereiro, R

Periasamy, AP

Peters, $T$

Petersen, E

Pethig, R

Petruci, JF

Phelan, M

Philipp, R

Phillips, MM

Phinney, KW

Pichon, V

Picó, Y

Piestansky, J

Pingarrón, J

Pino, $\mathrm{V}$

Piot, O
Pistiki, A

Pitt, B

Place, B

Plavan, G

Polo, F

Popov, AM

Popp, J

Posthuma-Trumpie, GA

Potthast, A

Prakash, M

Premasiri, R

Prentice, B

Priego-Capote, F

Primpke, S

Proefrock, D

Prohaska, T

Proll, G

Psillakis, E

$\mathrm{Pu}, \mathrm{K}$

Purcaro, G

Pursch, M

Qian, K

Qin, L

Qiu, B

Qiu, H

Qiu, J

$\mathrm{Qu}, \mathrm{F}$

Qu, L-L

Queiroz, MEC

Quintas, G

Quirino, JP

Rabin, I

Race, AM

Raftery, DM

Raghavan, S

Rajabi, HR

Ramanavicius, A

Ramineni, CK

Ramos, RM

Rampler, E

Ray, $\mathrm{T}$

Regal, P

Rehm, S

Reiner, JL

Ren, J

Ren, K

Ren, $\mathrm{Y}$

Renger, B

Renner, G

Resano, N

Reschiglian, $\mathrm{P}$ 
Reubsaet, L

Reyzer, ML

Ribeiro, AR

Ricci, M

Richon, D

Richter, R

Ridge, CD

Riegraf, C

Rios-Castro, A

Ripple, D

Ritvanen, T

Rizzo, F

Robb, $\mathrm{P}$

Rödiger, $\mathrm{S}$

Rodríguez Martín-Doimeadios, RC

Rodushkin, I

Rogers, $\mathrm{S}$

Rong, G

Ros, A

Rosati, G

Roshal, A

Rosi, F

Ross, A

Roth, G

Rout, CS

Roy, D

Roy-Lachapelle, A

Rubio Bravo, S

Rurack, K

Rusling, J

Rutledge, DN

Ryzhov, V

Saba, A

Sabbatini, L

Saeed, A

Sage, E

Sahore, V

Saito, Y

Šala, N

Salihovic, S

Salomone, A

Salvador, J-P

Salzer, R

Samal, SK

Samanidou, V

Sanda, M

Sander, LC

Sandra, M

Sarkar, HS

Sarma, N

Sarvin, B

Šatínský, D
Savina, MR

Sayyadi, N

Schantz, M

Schiel, J

Schirhagl, R

Schmid, R

Schmidt, TC

Schmitz, OJ

Schneider, RJ

Schönberger, T

Schug, KA

Schulze, M

Schymanski, EL

Segundo, MA

Sekhosana, KS

Sellergren, B

Sergi, M

Sethi, M

Sezgintürk, MK

Shaikh, M

Shajahan, A

Shamsi, MH

Shang, L

Shangguan, D

Sharma, A

Shaterian, N

Shaw, KJ

Sheta, SM

Shetti, NP

Shi, F

Shi, H

Shi, RL

Shih, S

Shiku, H

Shimizu, Y

Shimma, S

Shippy, S

Shiu, S

Shizuma, M

Shuang, S

Shuford, CM

Siegel, T

Sielemann, S

Sikes, HD

Sikirzhytski, V

Siluk, D

Singh, DR

Singh, JP

Sinha Roy, K

Sisco, E

Sivakumar, M

Skirtach, A

Skopp, G
Smith, E

Snel, MF

Snow, NH

Snozek, C

Snyder, NW

Soltwisch, J

Song, Y-Y

Soylak, M

Speck, P

Spence, DM

Spotto, G

Steiner, G

Steinwand, M

Stobiecka, M

Stocks, BB

Stove, CP

Strobbia, P

Struwe, W

Stubbs, S

Sturgen, R

Stürup, S

Styrishave, B

Su, X

$\mathrm{Su}, \mathrm{Z}$

Suder, PO

Sugimoto, I

Sun, J

Sun, L

Sun, S

Sundaresan, V

Supekar, NT

Suter, MJ-F

Sutton, AT

Svahn, O

Szostak, R

Szumski, M

Tagarelli, A

Takenaka, S

Takors, R

Talhout, R

Tam, M

Tamer, U

Tan, Y

Tang, B

Tang, D

Tang, K

Tang, L

Tanno, T

Tasic, L

Tata, A

Tavakkoli, N

Teo, A 
Tesařová, E

Tesch, MF

Thaysen-Andersen, M

Thevis, M

Thomaidis, NS

Thomas, KM

Thompson, $\mathrm{T}$

Tian, Z

Tittlemier, SA

Tok, TT

Tölgyesi, Á

Tombelli, S

Torsi, L

Torta, F

Touboul, D

Trabik, Y

Trammell, Z

Tranchida, PQ

Trim, PJ

Tripathi, KM

Trtic-Petrovic, TM

Trujillo-Rodríguez, MJ

Tsochatzis, ED

Tuccitto, N

Tuncel, A

Turiel, E

Turner, N

Turnipseed, SB

Ugarova, NN

Ulmer, CZ

Ul'yanovskii, NV

Urraca, JL

Vachet, RW

Valenti, G

Valentine, SJ

van de Merbel, N

van Harskamp, D

van Nuffel, S

Van Schepdael, A

Vanhaecke, F

Vanna, R

Vanninen, $\mathrm{P}$

Vardaki, M

Vashist, SK

Vasilescu, A

Vassileva, E

Vautz, W

Veenaas, C

Veeravalli, V

Veith, L

Venter, A
Verbeeck, N

Verona, E

Vetter, W

Viallon, J

Villalonga, R

Vogel, N

Voitechovic, E

Volk, DE

von Vacano, B

Vrzal, T

Vuckovic, D

Waddell Smith, D

Wagner, M

Walker, M

Wallace, MAG

Walt, D

Wan, J-B

Wang, A-J

Wang, BM

Wang, F

Wang, $\mathrm{H}$

Wang, H-B

Wang, H-S

Wang, J

Wang, L

Wang, M

Wang, R

Wang, S

Wang, W

Wang, Y

Wang, Z

Wang, ZZ

Warner, I

Waseem, A

Wasik, A

Weber, G

Wegscheider, W

Wei, G

Wei, H

Wei, W

Weimar, U

Weiss, V

Weller, MG

Weller, $\mathrm{P}$

Wen, Y

Weng, L-T

Wernisch, S

West, C

Whelan, RJ

White, F

Wieland, $\mathrm{K}$

Wietecha-Posłuszny, R
Wilkinson, $\mathrm{K}$

Wilson, D

Wilson, KR

Wilson, WB

Wisniewski, J

Witwer, KW

Wojcik, R

Wolfbeis, OS

Wong, SHY

Wongkaew, N

Workman, WE

Wright, C

Wrobel, K

$\mathrm{Wu}, \mathrm{J}$

$\mathrm{Wu}, \mathrm{K}$

$\mathrm{Wu}, \mathrm{R}$

Wu, S

$\mathrm{Wu}, \mathrm{Z}$

Wu, Z-Y

Wuethrich, A

Wunnapuk, K

Xia, Y

Xiang, $\mathrm{H}$

Xianghong, W

Xiao, $\mathrm{H}$

Xiao, Y

Xiaobo, Z

Xie, B

Xie, J

Xie, Q

Xie, Y

$\mathrm{Xu}, \mathrm{G}$

$\mathrm{Xu}, \mathrm{J}$

$\mathrm{Xu}, \mathrm{K}$

$\mathrm{Xu}, \mathrm{L}$

$\mathrm{Xu}, \mathrm{T}$

$\mathrm{Xu}, \mathrm{W}$

$\mathrm{Xu}, \mathrm{Y}$

$\mathrm{Xu}, \mathrm{Z}$

Xuan, X

Xue, M

Xue, R

Yagati, A

Yan, X

Yañez-Sedeño Orive, $\mathrm{P}$

Yang, CJ

Yang, C-S

Yang, $\mathrm{H}$

Yang, J

Yang, L

Yang, S 
Yang, W

Yang, Z

Yangcheng, L

Yao, W

Yao, Y

Ye, F

Ye, M

Ye, Y

Yi, F

$\mathrm{Yi}, \mathrm{L}$

Yin, $\mathrm{C}$

Yin, $\mathrm{X}-\mathrm{B}$

Yoon, HC

Yu, C-J

$\mathrm{Yu}, \mathrm{D}$

$\mathrm{Yu}, \mathrm{F}$

Yu, LL

$\mathrm{Yu}, \mathrm{M}$

Yu, T

$\mathrm{Yu}, \mathrm{Y}-\mathrm{L}$

Yuan, B

Yuan, R
Zabetakis, D

Zanella, R

Zarrine-Afsar, A

Zelada-Guillen, G

Zeleny, R

Zenkina, OV

Zestos, A

Zha, Z

Zhang, B

Zhang, H

Zhang, J

Zhang, J-K

Zhang, K

Zhang, L

Zhang, Q

Zhang, R

Zhang, S

Zhang, W

Zhang, X

Zhang, Y

Zhao, A

Zhao, H
Zhao, Q

Zhao, W

Zhao, Z

Zheng, G-X

Zhong, S

Zhong, W

Zhou, N

Zhou, Q

Zhou, W

Zhou, X

Zhou, Y

Zhou, ZS

Zhu, F

Zhu, J

Zhu, Q

Zhu, S

Zhuo, S

Zhuo, Y

Zimmermann, S

Zoccali, M

Zou, G

Züchner, T

Publisher's note Springer Nature remains neutral with regard to jurisdictional claims in published maps and institutional affiliations. 